\title{
Administración educativa. Plan de desarrollo de carrera para docentes en servicio
}

\author{
Educative administration. Career development plan for teachers in service
}

\author{
Eliécer Hernández \\ eliecerh81@gmail.com \\ Código ORCID: 0000000200811685 \\ Universidad Pedagógica Experimental Libertador- Miranda. Venezuela
}

- Artículo recibido en abril 2020

- Arbitrado en mayo 2020

RESUMEN

- Publicado en julio 2020

La finalidad del presente trabajo fue diseñar un plan de desarrollo de carrera para docentes en servicio de Categoría IV, V y VI en el área de administración educativa para la Unidad Educativa Nacional "José Avalos" de la parroquia El Valle, municipio bolivariano Libertador del Distrito Capital. El estudio fue de campo, el nivel descriptivo y la modalidad proyecto factible. El instrumento utilizado fue el cuestionario aplicado a veintisiete (27) docentes. Entre los resultados obtenidos está el hecho de que los docentes no han recibido formación en el área de administración educativa y que el personal directivo maneja débilmente las herramientas teórico-metodológicas para el abordaje de los temas en dicha área. Todo esto propició el diseño del plan de desarrollo de la carrera docente en el área de administración educativa, con el de brindar el apoyo necesario a los docentes en servicio.

Palabras clave: Plan de desarrollo; carrera docente; Administración Educativa

\section{ABSTRACT}

The purpose of this work was to design a career development plan for teachers in service of Category IV, V and VI in the area of educational administration for the National Educational Unit "José Avalos" of the parish El Valle, Bolivarian municipality Libertador District Capital. The study was field, descriptive level and feasible project modality. The instrument used was the questionnaire applied to twenty-seven (27) teachers. Among the results obtained is the fact that teachers have not received training in the area of educational administration and that the management staff weakly manages the theoretical-methodological tools for addressing the issues in that area. All this led to the design of the development plan for the teaching career in the area of educational administration, with the need to provide the necessary support to the teachers in service.

Keywords: Development plan; teaching career; Educational Administration 


\section{INTRODUCCIÓN}

La educación se asume como un elemento relevante en virtud de los cambios que se están dando en el orden científico, técnico y social, y que inciden directamente en el desarrollo socioeconómico de los países, lo que determina la necesidad de que los docentes respondan a las exigencias que implica el saber y el desarrollo de la ciencia, ya que él representa a un servidor público y su función es la administración del currículo como ejercicio docente; en este sentido, vale la pena destacar el planteamiento formulado por la Organización de Estados Iberoamericanos para la Educación, la Ciencia y la Cultura (2003), en el cual afirman que:

Asistimos a un replanteamiento del ejercicio docente, de las nuevas tareas de la enseñanza y de las implicaciones que tales revisiones tienen en el campo de la formación permanente del profesorado. Este hecho se vincula a los cambios profundos que se están produciendo en diversos terrenos, que ponen en cuestión las propias bases de la escuela y de la formación y práctica de maestros y profesores. (s.p)

Es así como las instituciones educativas se conciben como un espacio de gran relevancia social, donde el aprender se convierte en un elemento de desarrollo cultural, del proceso de expansión natural del conocimiento, y el desbordamiento hacia las fronteras del saber, como producto de una actividad académica que sea excelente en la medida en que se logre la vinculación entre sus miembros y su contexto.

De allí que es necesario definir el tipo de docente que requiere la sociedad, lo cual implica en palabras de Sánchez y Pérez (2005) "...la formulación de las políticas que orientan estas reformas se plantea la necesidad de contar con el docente ideal, en su aplicación no se establecen los criterios para formarlo" (pp. 140-141)

Es a partir de lo anterior que el docente es clave en una organización para el logro de los procesos educativos, y por su primacía surge el plan de desarrollo de carrera docente como el mecanismo para que los docentes adquieran herramientas y conocimientos en el área de administración educativa. Es por ello que para el desarrollo de esta investigación se asume el plan de desarrollo de carrera tal cual lo establece el Ministerio de Educación Cultura y Deportes (2003) al expresar:

La Dirección de Formación Permanente y Supervisión del MECD diseña las Políticas de Formación Permanente enfocándolas como un proceso continuo, que se inicia desde la gestación y trasciende al proceso de escolarización, implicando aspectos extraescolares. Su finalidad es lograr la formación de los docentes y de todos los miembros del sector 
educativo, además de la familia y la sociedad. Para ello, enmarca su Política en dos líneas estratégicas: (a) La Formación de todo el personal que integra el sector educativo: docentes, profesionales corresponsables del proceso educativo, así como el personal administrativo y obrero, por medio de los programas de evaluación institucional, investigación educativa y desarrollo profesional. Este último, contempla la formación inicial y la formación continua. (p. 44)

En coherencia con la idea anterior es preciso usar el plan de desarrollo de carrera docente como un conjunto de acciones programáticas que se le brinda al docente en ejercicio para tener herramientas y conocimientos sobre las diferentes áreas y cumplir así su función docente, y de esta manera fomentar la mejora continua de la praxis en el aula, y así dar respuesta a las necesidades tanto del alumno y de la sociedad marcado por el avance continuo del conocimiento.

Dentro del contexto descrito, se evidencia que la adquisición, habilidades, destrezas y conocimientos deben ser puntos de interés de quienes planifican las políticas educativas-, razón por la cual el marco de la educación y el perfil del docente debe armonizar la capacidad pedagógica con la vocación investigativa $\mathrm{y}$ con su proyecto social, factor que se constituye en el fundamento para la construcción de comunidades académicas, y con nuevas formas de organización y gestión, orientadas al logro de altos niveles de excelencia y calidad institucional.

La finalidad que se pretende es aportar ideas que puedan ser utilizadas por los entes que se ocupan de elaborar las políticas y los programas dirigidos al desarrollo profesional de los docentes que sean cónsonos con la realidad y den respuesta a las necesidades del sistema educativo.

Los planteamientos anteriores sirven de soporte para considerar la necesidad de proponer nuevos mecanismos que traducidos en modelos que puedan optimizar la actualización de los docentes, como respuesta a las exigencias de una gestión docente de calidad y con pertinencia a las nuevas realidades que presenta el sistema educativo bolivariano, tal como lo esboza Imbernón (1994) "engloba los procesos que mejoran el conocimiento profesional, las habilidades y las actitudes de los trabajadores de un centro educativo, por lo tanto, afecta a los equipos de gestión, al personal no docente y al profesorado" (p. 45)

En función de lo previo, cuando se habla de un plan de desarrollo de la carrera docente se piensa en un profesor que se encuentra ya en pleno ejercicio profesional, lo cual exige centrar la atención en el tema a través de "un posible acercamiento al concepto de desarrollo profesional del profesorado puede darse a través del proceso de mejora de los conocimientos 
profesionales" (Imbernón, 1994, p. 45), ya que se hace necesario reconocer la actitud por parte del docente en ejercicio para mantener el empeño en aprender de manera constante, en especial lo relativo a la institución educativa donde labora.

Es por ello que el Vicerrectorado de Docencia de la Universidad Pedagógica Experimental Libertador (UPEL) en el año 2006 propuso un Programa de Formación Nacional de Educación Media en relación con la Formación Docente para Educación Media, Diversificada y sobre la base de dos principios fundamentales determinados a partir de formar profesionales competitivos dentro y fuera del contexto nacional y promover la creatividad, la innovación y la educación permanente.

En cuanto al segundo principio el documento señala "la necesidad imperiosa de desarrollar la creatividad y la innovación en los alumnos" (p. 15). Ambos implican, como dice Laffitte citado por Sánchez (s.f):

el concepto de formación docente es más amplio que el de formación permanente. Debe entenderse como un proceso planificado, de crecimiento y mejora, en relación con el propio conocimiento, con las actitudes hacia el trabajo, con la institución, y buscando la interrelación entre las necesidades de desarrollo personal y las de desarrollo institucional y social. (s.p)
El plan de desarrollo de la carrera docente es la oportunidad de generar espacios en los cuales se pueda aprovechar los conocimientos tanto teórico como prácticos que ya el docente de cada área posee, propiciando el intercambio de ideas, propulsando el interés por la investigación, la adquisición de conocimientos en el manejo de la tecnología, la información y la comunicación; lo cual puede redundar en la construcción de la identidad profesional, que pretende el aumento de la satisfacción en el ejercicio de la profesión a través de una mayor comprensión y mejora de la competencia profesional, mediante el mejoramiento de sus habilidades y destrezas en el área laboral en la cual se desempeña.

En función de lo anterior Vezub (2007) señala que "a la hora de pensar las innovaciones pedagógicas y las políticas educativas ya nadie pasa por alto la problemática del docente y la calidad de su formación" (p. 2); todo lo cual indica que cualquier propuesta $o$ modelo de desarrollo de la carrera docente debe tener como epicentro al docente como beneficiario, pero al mismo tiempo la pieza clave de toda organización: el talento humano.

De lo antes expuesto, se aprecia un desfase de la realidad generada por el hecho que los planificadores piensan en los cambios deseados para el sistema educativo, dejando de lado a los docentes que son la pieza fundamental de la 
organización, tal como se evidencia en el Proyecto Educativo Nacional (1999) "los planes y programas de capacitación han sido diseñados sin tomar en cuenta las necesidades, experiencias y aprendizajes adquiridos de la práctica pedagógica y social del docente" (p. 56), y al hacer la instrumentación de los cambios establecidos por el órgano rector se encuentra con la realidad que los docentes no han sido formados para asumir los retos profesionales asociados a cualquier reforma en materia educativa.

Se considera que el docente que se encuentra activo pueda fortalecer su praxis pedagógica a través de la aplicación de un programa de formación en el área de Administración Educativa que lo lleve hacia los nuevos senderos del conocimiento, y así elevar su rendimiento profesional en las distintas áreas de trabajo como son administrativas, docentes y de investigación.

De acuerdo con lo antes señalado surgen las siguientes interrogantes, con miras a dar una respuesta al problema de investigación.

1. ¿Cuáles son las iniciativas por parte del Ministerio del Poder Popular para la Educación en materia de Desarrollo de Carrera Docente para Educación Media General?

2. ¿Cuáles son los criterios a utilizar para elaborar un plan de desarrollo de carrera para los docentes de categoría IV, V y VI de la Unidad Educativa
Nacional "José Avalos" de la Parroquia E1 Valle Municipio Bolivariano Libertador del Distrito Capital?

3. ¿Cuáles son los contenidos programáticos y estrategias metodológicas del plan de desarrollo de carrera para los docentes de categoría IV, V y VI en el área de Administración Educativa para la Unidad Educativa Nacional "José Avalos" de la Parroquia El Valle Municipio Bolivariano Libertador del Distrito Capital?

\section{Objetivos de la investigación}

\section{Objetivo general:}

Diseñar un Plan de Desarrollo de Carrera para Docentes en Servicio De Categoría IV, V y VI en el Área de Administración Educativa para la Unidad Educativa Nacional "José Avalos" de la Parroquia El Valle Municipio Bolivariano Libertador Del Distrito Capital.

\section{Objetivos específicos:}

1. Diagnosticar las iniciativas por parte del Ministerio del Poder Popular para la Educación en materia de Desarrollo de Carrera Docente para Educación Media General.

2. Describir los criterios a utilizar para elaborar un plan de desarrollo de carrera para los docentes de categoría IV, V y VI de la Unidad Educativa Nacional "José Avalos" de la 
Parroquia El Valle Municipio Bolivariano Libertador del Distrito Capital.

3. Determinar los contenidos programáticos y estrategias metodológicas del plan de desarrollo de carrera para los docentes de categoría IV, V y VI en el área de Administración Educativa para la Unidad Educativa Nacional "José Avalos" de la Parroquia El Valle Municipio Bolivariano Libertador del Distrito Capital.

\section{MÉTODO}

El diseño de la investigación fue no experimental ya que este no contempla la manipulación intencional de las variables de estudio, y el tipo investigación fue de campo debido a que los datos fueron tomados directamente de la realidad objeto de estudio.

La muestra estuvo representada por 27 profesores de la Unidad Educativa Nacional "José Avalos" de la parroquia El Valle, municipio bolivariano Libertador del Distrito Capital de acuerdo con los siguientes criterios:

1. Que posean la titularidad del cargo que desempeña

2. Que este clasificado la categoría IV, V y VI.

3. Que tengan dedicación de tiempo completo.
La técnica que se utilizó fue la encuesta y se aplicó a la muestra un cuestionario.

\section{RESULTADOS}

En relación con el objetivo específico $\mathrm{N}^{\circ} 1$ que expresa: diagnosticar las iniciativas por parte del Ministerio del Poder Popular para la Educación en materia de desarrollo de carrera docente para Educación Media General se realizaron tablas de frecuencia a través del uso de la estadística descriptiva; el diagnóstico se llevó a cabo a través de la observación participante ya que el investigador al momento del estudio se desempeñaba como profesor por horas en la institución, dichas observaciones fueron recogidas a través una hoja de anotaciones como producto de encuentros informales con la población objeto de estudio.

Para realizar el análisis de la variable Iniciativas de formación docente, se estudiaron los 5 primeros ítems del cuestionario, tal como se muestra a continuación.

En relación con el ítem número uno (1) que expresa si se promueven talleres en la Unidad Educativa Nacional "José Avalos" de la Parroquia El Valle Municipio Bolivariano Libertador del Distrito Capital, se puede afirmar que un $77,8 \%$ considera que "nunca" se realizan talleres, mientras que el $22,2 \%$ reconoce que "a veces" ha recibido 
algún tipo de atención para su desarrollo llevada a cabo por el Ministerio del profesional. De acuerdo a los resultados Poder Popular para la Educación en la obtenidos, es posible deducir que la institución educativa seleccionada para promoción de talleres sobre efectuar el estudio (ver Tabla 1 y Administración Educativa, rara vez es Gráfico 1).

Tabla 1. Ítem 1.- El MPPE promueve en la UEN José Avalos talleres sobre administración educativa para los docentes

\begin{tabular}{llll}
\hline Frecuencia & & Porcentaje & Porcentaje acumulado \\
\hline Nunca & 21 & 77,8 & 77,8 \\
A veces & 6 & 22,2 & 100,0 \\
Casi Siempre & 0 & 0,00 & 0,00 \\
Siempre & 0 & 0,00 & 0,00 \\
Total & 27 & 100 & 100 \\
\hline
\end{tabular}

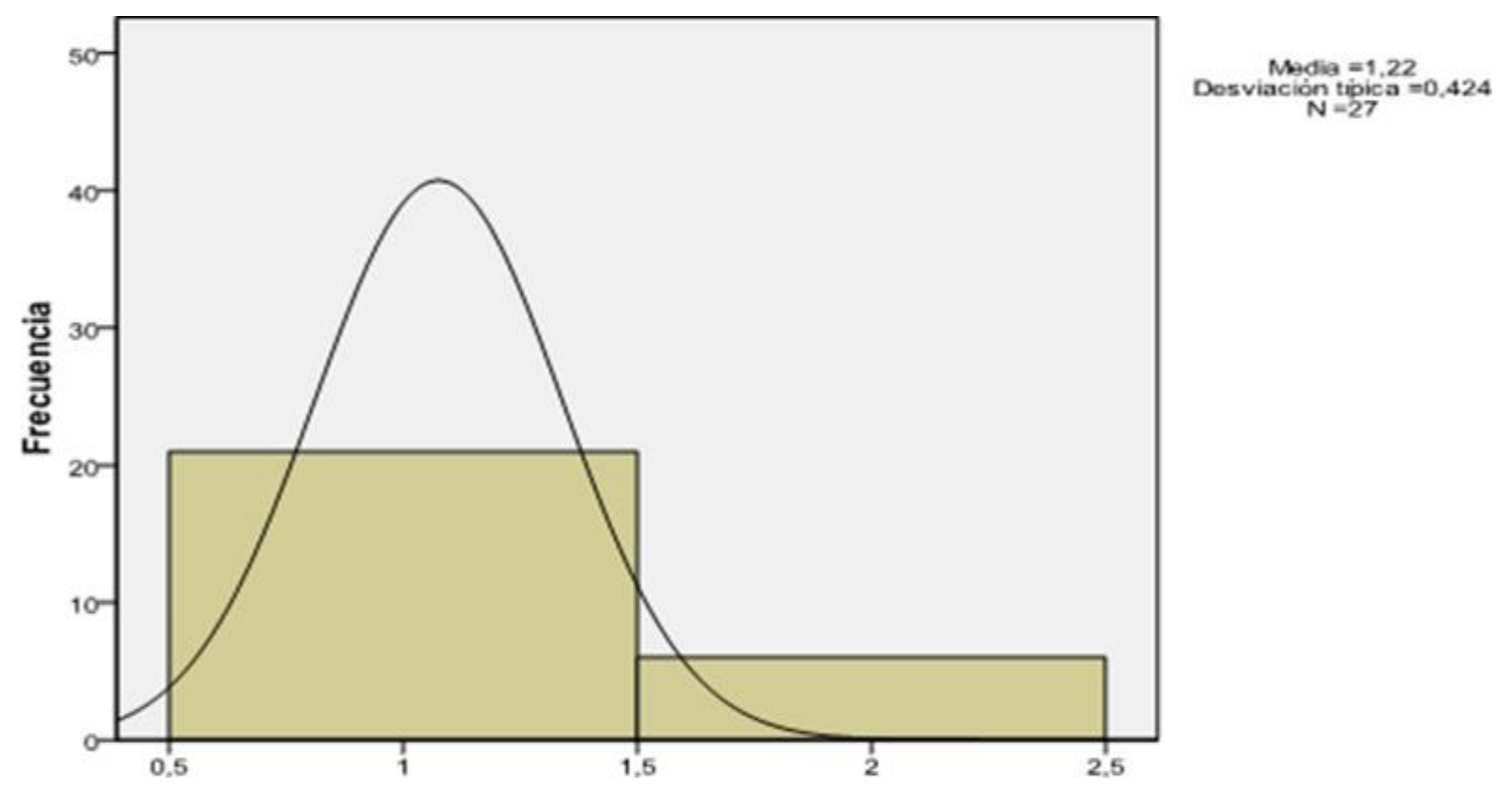

Gráfico 1. Resultado Ítem 1 
Se valida la necesidad de dar respuesta a la problemática gerencial que permita atender las necesidades en términos de recursos humanos en el manejo de la nómina y así propulsar el desarrollo de la carrera docente en aras de elevar la eficiencia gerencial y académica de la Unidad Educativa Nacional "José Avalos" de la Parroquia El Valle Municipio Bolivariano Libertador del Distrito Capital.

En cuanto al objetivo $\mathrm{N}^{\circ}$ dos (2) que expresa, describir los criterios a utilizar para un plan de desarrollo de carrera para los docentes de categoría IV, V y VI de la Unidad Educativa Nacional "José Avalos" de la Parroquia El Valle Municipio Bolivariano Libertador del Distrito Capital.

En el ítem número seis (6) que expresa si se realizan reuniones del personal directivo con los docentes de la Unidad Educativa Nacional "José Avalos" de la parroquia El Valle, municipio bolivariano Libertador del Distrito Capital, se puede afirmar que un $33,38 \%$ considera que "a veces" se realizan reuniones, mientras que el $55,6 \%$ reconoce que "casi siempre" se efectúan reuniones con el personal y un $11,1 \%$ reconoce que "siempre" se llevan a cabo las reuniones. De acuerdo a los resultados obtenidos, es posible deducir que la celebración de reuniones entre el personal directivo y docente se realiza "casi siempre"; lo cual hace deducir que con regularidad se producen estos encuentros de carácter técnico para afinar detalles vinculados a la programación académica. (ver Tabla 2 y Gráfico 2)

Tabla 2. Ítem 6.- En la U.E.N. "José Avalos" el personal directivo efectúa reuniones con el personal docente

\begin{tabular}{llll}
\hline Frecuencia & & Porcentaje & Porcentaje acumulado \\
\hline Nunca & 9 & 33,3 & 33,3 \\
A veces & 15 & 55,6 & 88,9 \\
Casi Siempre & 0 & 0,00 & 0,00 \\
Siempre & 3 & 11,1 & 100,00 \\
Total & 27 & 100 & 100 \\
\hline
\end{tabular}




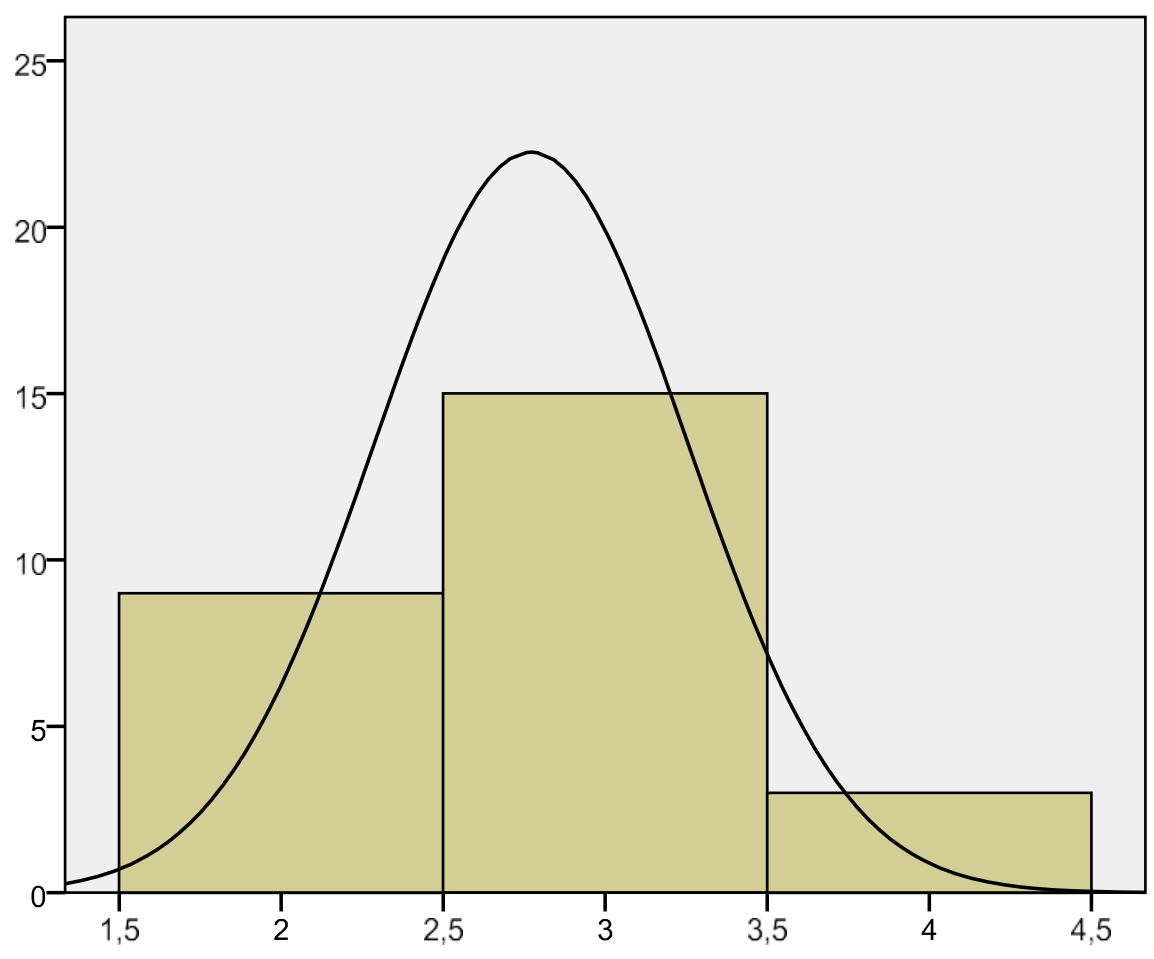

Gráfico 2. Ítem 6

En contexto con los resultados obtenidos, los miembros del personal directivo reúnen al personal docente como una forma de transmitir de manera directa los lineamientos inherentes al trabajo pedagógico y administrativo a ejecutar en cada uno de los períodos académicos.

La realización de reuniones en la U.E.N "José Avalos" abre un espacio de intercambio de ideas entre todos los miembros del personal docente para socializar las ideas y experiencias en favor de cumplir los objetivos planteados en los diferentes proyectos elaborados para cada año escolar, y al mismo tiempo estar en sintonía con los lineamientos provenientes del Ministerio del Poder Popular para la Educación,
Zona Educativa del Distrito Capital y el Distrito Escolar N ${ }^{\circ} 1$.

En cuanto al objetivo $\mathrm{N}^{\circ} 3$ (tres) que expresa, determinar los contenidos programáticos y estrategias metodológicas del plan de desarrollo de carrera para los docentes de categoría IV, V y VI en el área de Administración Educativa para la Unidad Educativa Nacional "José Avalos" de la Parroquia El Valle Municipio Bolivariano Libertador del Distrito Capital, se estudiaron los 03 ítems del cuestionario, tal como se muestra a continuación:

Los resultados obtenidos del ítem número dieciséis (16) permiten evidenciar que en el caso de las respuestas aportadas por los consultados a través del cuestionario son un $74,1 \%$ 
para la respuesta identificada como nunca, mientras que un $25,9 \%$ se inclinó por la respuesta denominada "a veces". En función de los datos se puede deducir con tono objetivo que en la U.E.N.
"José Avalos" el personal se ve imposibilitado de tener acceso a un programa de desarrollo profesional en particular de Administración Educativa (ver Tabla 3 y Gráfico 3).

Tabla 3. Ítem 16.- En la UEN José Avalos el docente tiene acceso a un plan de desarrollo de la carrera docente con base en la Administración Educativa

\begin{tabular}{lccc}
\hline & Frecuencia & Porcentaje & Porcentaje acumulado \\
\hline Nunca & 20 & 74,1 & 74,1 \\
A veces & 7 & 25,9 & 100,0 \\
Casi Siempre & 0 & 0,0 & 100,0 \\
Siempre & 0 & 0,0 & 100,0 \\
Total & 27 & 100,0 & \\
\hline
\end{tabular}

Gráfico 3. Ítem 16

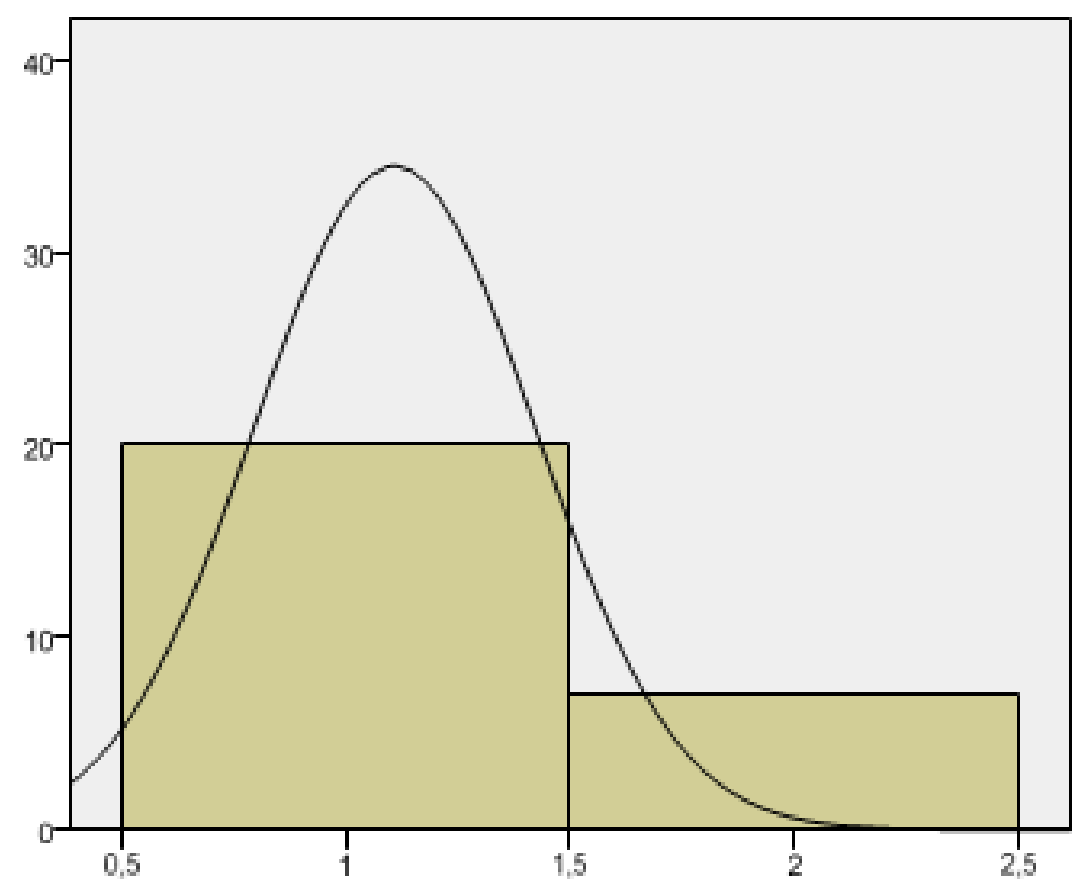

Los resultados obtenidos permiten validar la necesidad de brindar el apoyo necesario a los profesionales de la docencia para acceder a un plan de desarrollo de la carrera docente con énfasis en el área de la administración educativa como mecanismo para acceder a herramientas teórico metodológicas que favorezcan su crecimiento en el desempeño de sus labores docentes y administrativas. 


\section{Propuesta}

Plan de Desarrollo de carrera para docentes en servicio de categoría IV, $\mathrm{V}$ Y VI en el área de Administración educativa

\section{Fundamentación}

En la actualidad los planes de desarrollo para el docente es una vía que le puede estimular y crear los mecanismos adecuados para el desarrollo de su carrera y al mismo tiempo fomentar un conjunto de saberes que le permitirán abordar temas de carácter académico y científico, de acuerdo a la realidad concreta de cada institución.

El desarrollo docente es clave ya que da acceso a conocimientos, competencias y habilidades que luego son de importancia capital toda vez que asumida su carrera profesional. En correspondencia con las ideas expuestas se hace necesario rescatar y mejorar la calidad de la formación y de la práctica docente; dentro del marco de esta realidad surge la intención de la propuesta ya que se plantea: diseñar un plan de desarrollo de carrera para docentes en servicio de categoría IV, V y VI en el área de administración educativa para la U.E.N. "José Avalos" de la Parroquia El Valle Municipio Bolivariano Libertador del Distrito Capital.

\section{Propósito}

a) Propiciar el intercambio de ideas por parte de los docentes para lograr la cohesión grupal con miras a su desarrollo personal y académico.

b) Promover en los docentes la responsabilidad con respecto al desarrollo de su carrera docente durante su presencia como docente activo de la institución.

c) Conocer las normas y procedimientos de tipo docente y administrativo que se llevan a cabo en la institución.

d) Estimular en los docentes la cultura de la institución y la puesta en práctica de la misma en favor del sentido de pertenencia.

e) Implementar el plan de desarrollo de la carrera docente dirigido a mejorar su trabajo docente y administrativo en la institución.

\section{Objetivos}

El Plan de Desarrollo de la carrera docente tiene como objetivo el promover la administración educativa como elemento de desarrollo del personal docente de la Unidad Educativa Nacional "José Avalos" de la Parroquia El Valle Municipio Bolivariano Libertador del Distrito Capital. En concordancia con el planteamiento anterior, son objetivos integradores del Plan de desarrollo, los siguientes: 


\section{Objetivos integradores}

1. Conocer los aspectos teóricos de la administración educativa.

2. Identificar la responsabilidad en establecimientos educativos: públicos y privados.
3. Identificar las herramientas para la administración educativa.

4. Promover administración educativa como elemento de desarrollo profesional.

METODOLOGÍA: se organizará el plan de desarrollo de la carrera docente a partir de la figura del taller. Se dividirá a los participantes en tres grupos para discutir el material bibliográfico, luego se socializará en una plenaria donde se le proyectará un video sobre los diferentes tópicos a tratar.

MATERIAL ENTREGADO: se entregará una carpeta con todo el material referido a la administración educativa y que contiene lecturas referidas de acuerdo al cronograma de actividades. 
Talleres para llevar a cabo el Plan de desarrollo de la Carrera Docente

\begin{tabular}{|c|c|c|c|}
\hline Temas & Contenidos & Estrategias Instruccionales & Estrategias de Evaluación \\
\hline La administración educativa & $\begin{array}{l}\text { 1. Concepto de Administración } \\
\text { Educativa. } \\
\text { 2. Campos de la Administración } \\
\text { Educativa. } \\
\text { 3. Objeto de la Administración } \\
\text { Educativa }\end{array}$ & $\begin{array}{l}\text { 1. Pre-instruccionales } \\
\text { 1. Este curso cuenta con lecturas con la } \\
\text { finalidad de lograr a través de } \\
\text { actividades comprender los objetivos } \\
\text { propuestos. }\end{array}$ & $\begin{array}{l}\text { Postinstruccionales formativa: } \\
\text { 1. Participa en las asesorías que } \\
\text { te ofrece el facilitador del } \\
\text { curso, para iniciar el proceso } \\
\text { de lecturas del material. }\end{array}$ \\
\hline $\begin{array}{l}\text { La responsabilidad en establecimientos } \\
\text { educativos: públicos y privados }\end{array}$ & $\begin{array}{l}\text { 1. La administración de la } \\
\text { educación pública y privada } \\
\text { 2. Contratos de trabajo }\end{array}$ & $\begin{array}{l}\text { 2. Antes de comenzar a estudiar los } \\
\text { contenidos de este curso, debes } \\
\text { realizar una lectura profunda de las } \\
\text { lecturas y focalizar las actividades de }\end{array}$ & $\begin{array}{l}\text { 2. Reúnete con un grupo de } \\
\text { trabajo para discutir las } \\
\text { lecturas y desarrollar las } \\
\text { actividades previstas para } \\
\text { cada objetivo del taller }\end{array}$ \\
\hline
\end{tabular}

3. Derechos y deberes del docente

4. Reformas educativas

1. Gestión Educativa.

Herramientas para la administración educativa

2. Gerencia Educativa

3. Documentos preparados por el

Ministerio Popular para la

Educación evaluación para luego comparar con

las actividades de auto evaluación.

\section{Co-Instruccionales}

3. Se recomienda organizar grupos de trabajo de 3 a 4 personas; la idea es propiciar el aprendizaje colaborativo.

\section{Orientaciones generales para el estudio de cada objetivo}

El contenido fundamental del taller está representado por las lecturas, por lo tanto el participante debe centrar su atención en los textos de los autores seleccionados y la bibliografía complementaria. Las Siguientes orientaciones le facilitara el aprendizaje: 
Talleres para llevar a cabo el Plan de desarrollo de la Carrera Docente

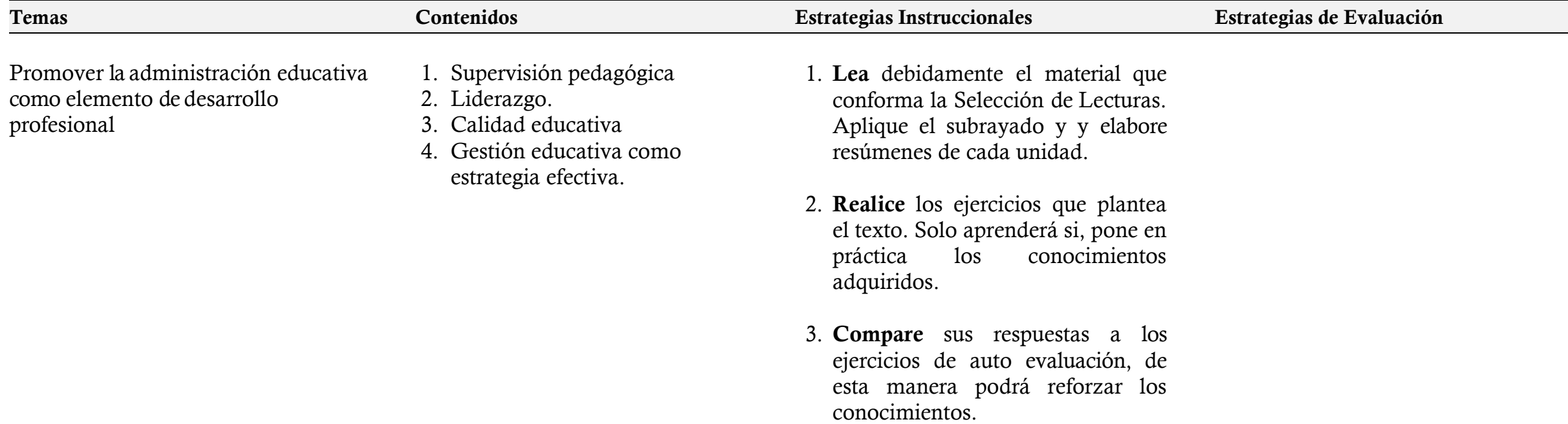

1. Supervisión pedagógica

. Liderazgo. 
FASE I: PROMOCIÓN

Promover la administración educativa como elemento de desarrollo profesional del personal docente de la Unidad Educativa Nacional "José Avalos" de la Parroquia El Valle Municipio Bolivariano Libertador del Distrito Capital.

Estrategias

Actividades

Técnicas

\section{Motivación Visual}

- Selección de contenidos para la diagramación, montaje y exhibición de carteleras informativas sobre:

- La administración educativa.

- La responsabilidad en establecimientos educativos: públicos y privados

- Herramientas para la administración educativa

- Promoción de la administración educativa como elemento de desarrollo profesional
- Lectura Informativa y reflexiva

- Concepto de administración educativa.

- Campos de la administración educativa.

- Objeto de la administración educativa

- La administración de la educación pública y privada

- Contratos de trabajo

- Derechos y deberes del docente

- Reformas educativas

- Gestión Educativa.

- Gerencia Educativa

- Documentos preparados por el Ministerio Popular para la Educación

- Supervisión pedagógica

- Liderazgo.

- Calidad educativa

- Gestión educativa como estrategia efectiva.
Recursos

Tiempo
- Bibliografía Especializada 1 Semana Cartelera

antes del evento
- Papel bond

- Afiches

- Marcadores Tijeras

- Bibliografía especializada

- Hoja Blanca tipo Carta

Desplegables a Color 


\section{FASE I: PROMOCIÓN}

Promover la administración educativa como elemento de desarrollo profesional del personal docente de la Unidad Educativa Nacional "José Avalos" de la Parroquia El Valle Municipio Bolivariano Libertador del Distrito Capital.

\section{Estrategias}

\section{Actividades}

- Desarrollo de dinámicas de Integración Grupal

Establecimiento de normas de funcionamiento.

Exposición de expectativas sobre el programa propuesto.

- Charlas informativas sobre las características importancia, dimensiones, y enfoque, del programa propuesto.

\section{Técnicas}

- Dinámica grupal

- Desarrollo de la dinámica y procesamiento.

- Discusión socializada

- Lluvia de ideas

- Discusión socializada

- Lluvia de ideas

- Reflexión

Exposición oral

- Reflexión

\section{Recurso}

Auditorio

Bibliografía Especializada

Dípticos

Trípticos Computadora

Portátil Video Beam Auditorio

Bibliografía Especializada

Computadora Portátil Video

Beam

Auditorio

\section{Tiempo}

\section{4 horas}

Exposición ora

Discusión socializada formación y contenidos del programa propuesto.

\section{Evaluación:}

Asistencia y participación en las actividades programadas (demostradas a través de conductas observables como: motivación, interés, atención, cambio de actitudes, interacciones grupales y responsabilidad) 


\section{Fase II: FORMACIÓN}

Objetivo. Promover la administración educativa como elemento de desarrollo del personal docente de la Unidad Educativa Nacional "José Avalos" de la Parroquia El Valle Municipio Bolivariano Libertador del Distrito Capital.

Objetivos específicos

\section{Conocer los aspectos teóricos de la administración}

educativa.

Identificar la responsabilidad en establecimientos

educativos públicos $\mathrm{y}$ privados.

\section{Contenidos}

1. Concepto de Administración Educativa.

2. Campos de la Administración Educativa.

3. Objeto de la Administración Educativa

1. La administración de la educación pública y privada

2. Contratos de trabajo

3. Derechos ydeberes del docente

4. Reformas educativas

\section{Estrategas metodológicas}

1. E1 facilitador da la bienvenida al taller

2. Cada participante se presentará en cadena citando una característica personal.

3. Participación espontánea de lo que esperan obtener en el taller.

4. E1 facilitador explicara sobre la importancia de las normas dentro de un grupo.

5. Se expresarán y escribirán las normas de funcionamiento del grupo.

1. E1 facilitador mostrará una presentación visual sobre los diferentes modelos de educación y posteriormente motivará a formar equipos de trabajo para una discusión socializada sobre los modelos presentados, para luego reflexionar sobre: La administración de la educación pública y privada

2. El facilitador invitara a realizar una ronda grupal para compartir lo discutido. Pedirá a cada participante que se identifique con uno de los modelos trabajados y el porqué de su identificación.

3. El facilitador expresara la importancia de Contratos de trabajo y Derechos y deberes del docente.

\section{Recursos}

Humanos:

Facilitador

Participantes

Materiales:

Información

fotocopiada. Papel

bond. Cartulinas.

Revistas. Hojas blancas.

Marcadores. Lápices

Bolígrafos. Espacio

físico.

Humanos: Facilitador y participantes. 


\section{Fase II: FORMACIÓN}

Objetivo. Promover la administración educativa como elemento de desarrollo del personal docente de la Unidad Educativa Nacional "José Avalos" de la Parroquia El Valle Municipio Bolivariano Libertador del Distrito Capital.

Objetivos específicos

Identificar las herramientas para la administración educativa.

\section{Promover administración educativa como Elemento de desarrollo profesional}

\section{Contenidos}

1. Gestión educativa

2. Gerencia educativa

3. Documentos preparados por el Ministerio Popular para la Educación
1. Supervisión pedagógica

2. Liderazgo.

3. Calidad educativa

4. Gestión Educativa como estrategia efectiva.

\section{Estrategas metodológicas}

1. Después que el facilitador exponga sobre Gestión y Gerencia educativa, invitara a formar tres grupos para planificar una dramatización donde se evidencien el dominio de los contenidos

2. Se invitará a cada grupo a presentar su dramatización, para luego realizar ronda de discusión socializada y reflexión. Se hará resumen del contenido y del proceso vivido en el taller.

3. El facilitador motivará para que los participantes expresen voluntariamente:

¿Qué aprendieron?

¿Se comprometen a llevar a la práctica lo aprendido?

¿Qué actividad o estrategia fue la más significativa?

1. Después de una breve presentación, se motivará a los participantes a responder la siguiente interrogante:

¿Te consideras líder? ¿Por qué?

2. Aprovechando las respuestas de los participantes se iniciará una discusión para socializar las respuestas.

3. El facilitador invitará a formar quintetos y entregará material relacionado a:

Roles del líder. Características del líder. Estilos de liderazgo docente.

4. Una vez analizado el material por los equipos de trabajo se realizará un torbellino de ideas donde los participantes verbalizaran las ideas del análisis realizado a la teoría, el facilitador

\section{Recursos}

Materiales:

Información fotocopiada. Papel

bond. Cartulinas.

Revistas. Hojas

blancas.

Marcadores

Lápices Bolígrafos

Espacio físico.

\section{Humanos:}

Facilitador y participantes

Materiales:

Información

fotocopiado

Papel bond 


\section{Fase II: Formación}

Objetivo. Promover la administración educativa como elemento de desarrollo del personal docente de la Unidad Educativa Nacional "José Avalos" de la Parroquia El Valle Municipio Bolivariano Libertador del Distrito Capital.

\section{Objetivos específicos}

\section{Contenidos}

\section{Estrategas metodológicas}

5. Aprovechará el momento de discusión para aclarar dudas y hacer los reforzamientos respectivos.

6. El facilitador motivará a los participantes para desarrollar la dinámica "Características de un líder", con la finalidad de comparar los resultados de una decisión individual con los de una decisión grupal y la de explorar los valores que caracterizan a un líder.

7. Luego de realizar la dinámica el facilitador preguntará:

¿Cómo se sintieron con la dinámica?

¿De qué se dieron cuenta?

¿Cuál es la importancia del liderazgo en la profesión docente?

8. Aprovechando las respuestas de los participantes se mostrará un video sobre: "El liderazgo en Educación"

9. Se invitará a los participantes a realizar una ronda para socializar sobre el contenido del video y reflexionar sobre las conductas de entrada y de salida.

10. Despedida.

\section{Recursos}

Cartulinas. Revistas

Hojas blancas.

Marcadores. Lápices.

Bolígrafos.

Espacio físico.

\section{Evaluación:}

Asistencia y participación en las actividades programadas (demostradas a través de conductas observables como: motivación, interés, atención, cambio de actitudes, interacciones grupales y responsabilidad) 


\section{CONCLUSIONES}

En relación con la interrogante acerca de ¿Cuáles son las iniciativas por parte del Ministerio del Poder Popular para la Educación en materia de desarrollo de carrera docente para Educación Media General?, se puede concluir que el ente rector en materia educativa no ha llevado a cabo el plan de desarrollo docente en la institución, razón por la cual es prudente dar respuesta ya que los cambios que ha generado la puesta en marcha la reforma educativa.

Sobre la interrogante: ¿Cuáles son los criterios a utilizar para elaborar un plan de desarrollo de carrera para los docentes de categoría IV, V y VI de la Unidad Educativa Nacional "José Avalos" de la parroquia El Valle, municipio bolivariano Libertador del Distrito Capital?, se pudo constatar que en los procesos que se llevan a cabo en la institución educativa se aplican tareas de tipo gerencial pero es preciso estar en consonancia con las políticas del Estado venezolano y los con las bases legales que rigen el sistema educativo; tomando en cuenta los documentos elaborados al respecto por el Ministerio del Poder Popular para la Educación en el marco del Liceo Bolivariano.

Sobre la pregunta ¿Cuáles son los contenidos programáticos y estrategias metodológicas del plan de desarrollo de carrera para los docentes de categoría IV, V y VI en el área de Administración
Educativa para la Unidad Educativa Nacional "José Avalos" de la parroquia El Valle, municipio bolivariano Libertador del Distrito Capital? En este punto se puede señalar que el área de Administración Educativa cobra fuerza debido a que los docentes que alcanzan las mencionadas categorías en el escalafón vigente en el Reglamento del Ejercicio de la Profesión Docente pueden aspirar y desempeñar cargos de tipo administrativo, de allí que el plan de desarrollo de la carrera docente exige la incorporación de temas gerenciales con el propósito de brindar herramientas de tipo teórico metodológicos a los docentes que le permitirán desempeñar con calidad las funciones que asuman en las dependencias de la institución.

\section{REFERENCIAS}

Galvis, R. V. (2006). Propuesta del programa nacional de educación media en relación con la formación del docente para educación media, diversificada y profesional. Recuperado de http://150.187. 142.20/info-general

/eventos/Pregrado/Archivos/Prop DoceMed.pdf

Imbernón, F. (1994). La formación y el desarrollo profesional del profesorado: Hacia una nueva cultura profesional. Barcelona: Biblioteca de aula

Ministerio de Educación, Dirección Sectorial de Planificación y Presupuesto. (1999, Octubre). Proyecto educativo nacional: Versión preliminar de la sistematización de las propuestas regionales. Caracas: Autor 
Ministerio de Educación Cultura y Deportes, Grupo Técnico del Plan Nacional de Educación para Todos. (2003, Agosto). Venezuela y la Educación para todos: Balance nacional y programa de trabajo Recuperado de http://planipolis.iiep.unesco.org/u pload/Venezuela/Venezuela\%20E FA\%20Plan.pdf

Sánchez Carreño, J. y Pérez Luna, E. (2005). Perspectivas actuales en la formación del docente latinoamericano .Integración Universitaria. 5(2)
Sánchez Nuñez, J. A. (s.f). E1 desarrollo profesional del docente universitario. Universidad Politécnica de Madrid. España. Recuperado de http://www.udual.org/CIDU/Rev ista/22/DesarrolloProfesional.htm Vezub, L. F. (2007). La formación y el desarrollo profesional docente frente a los nuevos desafíos de la escolaridad. Profesorado. 11(1). Recuperado de http://issuu.com /kikillo/docs/100629_iipe_el_desar rollo_prof_docente_centrado_ 